\title{
The value of early positive nucleic acid test and negative conversion time of SARS-CoV-2 RNA in the clinical outcome of COVID-19 patients
}

\section{Yongzhen Zhai ( $\square$ zyz200084@sina.com )}

Department of Infectious Disease, Shengjing Hospital of China Medical University https://orcid.org/0000-0002-7665-9683

\section{Xin Zang}

Department of Infectious Disease, Shengjing Hospital of China Medical University

\section{Liangkun Xiong}

Department of Hepatobiliary Surgery, Renmin Hospital of Wuhan University

Junyao Zhu

Department of Infectious Disease, Shengjing Hospital of China Medical University

\section{Fangfang Zhao}

Department of Infectious Disease, Fujian Medical University Affiliated First Quanzhou Hospital

\section{Shihong Wang}

Department of Paediatrics, Renmin Hospital of Wuhan University

\section{Wenhui Zeng}

Department of Hepatobiliary Surgery, Renmin Hospital of Wuhan University

\section{Kaihuan Yu}

Department of Hepatobiliary Surgery, Renmin Hospital of Wuhan University

\section{Research article}

Keywords: COVID-19, SARS-CoV-2, nucleic acid test, negative conversion, clinical characteristics

Posted Date: July 28th, 2021

DOI: https://doi.org/10.21203/rs.3.rs-753442/v1

License: (c) (i) This work is licensed under a Creative Commons Attribution 4.0 International License. Read Full License 


\section{Abstract \\ Background}

The outbreak of coronavirus disease (COVID-19) poses a great threat to the global public health. At present, the number of new confirmed COVID-19 cases and new deaths is increasing worldwide. The strategy of comprehensive and scientific detection of severe acute respiratory syndrome coronavirus 2 (SARS-CoV-2, the virus that causes COVID-19) through real-time reverse transcriptase polymerase chain

reaction (RT-PCR) for special populations and environments provides great support for the prevention and control of this pandemic in China. Our study focused on determining the factors associated with the length of time from symptom onset to the first positive nucleic acid test of throat swabs in COVID-19 patients, evaluating the effect of early positive nucleic acid detection on the disease severity and its significance in prognosis, and predicting the factors associated with the time from positive SARS-CoV-2 RNA test to negative conversion in COVID-19 patients.

\section{Methods}

This study included 116 hospitalized patients with COVID-19 from January 30, 2020 to March 4, 2020 in Wuhan, China. Throat swabs samples were collected for real-time reverse transcriptase polymerase chain reaction (RT-PCR) test of SARS-CoV-2 RNA, and all patients included in this study were positive for this test.

\section{Results}

Multivariate Cox proportional hazards model showed that disease severity and the duration of disease before admission to hospitals were protective factors for the time from symptom onset to positive nucleic acid detection, and the time from positive nucleic acid test to negative conversion was a risk factor for the time from symptom onset to positive nucleic acid detection. Meanwhile, the time from symptom onset to positive nucleic acid detection was an independent risk factor for the prolonged negative conversion of SARS-CoV-2 virus.

\section{Conclusions}

Patients with more severe disease and longer duration of disease before admission to hospitals had a shorter time from symptom onset to positive nucleic acid test. Prolonged time from symptom onset to positive nucleic acid test was an independent risk factor for the prolonged negative conversion time of SARS-CoV-2 virus, and the severity of the disease had no correlation with negative conversion time of SARS-CoV-2 virus. 


\section{Background}

Coronavirus disease (COVID-19) is a new infectious disease caused by a newly discovered coronavirus, severe acute respiratory syndrome coronavirus 2 (SARS-CoV-2). Since the outbreak in Wuhan in December 2019, the pandemic has spread to the world rapidly. At present, the number of new confirmed cases and new deaths is increasing worldwide. According to the World Health Organization (WHO), the number of new COVID-19 cases worldwide has been declining for seven consecutive weeks. However, the number of new deaths per day has not shown a clear downward trend. As of July 2, a total of 183 million cases of laboratory-confirmed SARS-CoV-2 infection and more than 3.96 million deaths were registered worldwide. Detection of SARS-CoV-2 included etiological and serological examination of this virus. Among the etiological examination methods, the detection of SARS-CoV-2 nucleic acid by reverse RT-PCR is still the gold standard for the diagnosis of COVID-19. Due to its capability of early diagnosis, high sensitivity and specificity, RT-PCR is widely used in the fields of suspected case diagnosis, population screening, and health monitoring of staff $(1,2)$. Two consecutive negative nucleic acid tests for respiratory pathogens (sampled at $\geq 24$ hours apart) are one of the important criteria to define a patient's recovery. Therefore, negative conversion of SARS-CoV-2 RNA is essential to confirming whether a patient meets the criteria for discharge. Previous studies have shown that advanced age, more comorbid underlying diseases, fever and corticosteroid therapy use, are risk factors for prolonged nucleic acid conversion(3-5). Recently, the emergence of new SARS-CoV-2 lineage B.1.617 has been associated with a surge in the number of infections in India(6). Mutations and recombination which lead to the emergence of novel lineages of SARS-CoV-2 can reduce the sensitivity of RT-qPCR and cause false negative results in throat swab detection(7). However, only a few studies have explored the factors influencing the length of time from the onset of symptoms to the first nucleic acid test positive and the value of early positive nucleic acid test on the clinical outcome of patients. In this study, we retrospectively assessed the clinical characteristics of mild and severe COVID-19 patients in Wuhan, explored the factors associated with the length of time from symptom onset to the first positive nucleic acid test of throat swabs and also investigated the risk factors for prolonged conversion time from positive to negative nucleic acid test, as well as the effect of early positive nucleic acid test on the severity of disease and its significance in prognosis of patients, so as to promote the early diagnosis and treatment of patients and improve the prognosis of patients.

\section{Materials And Methods}

\section{Study Design and subjects}

We retrospectively analyzed the clinical characteristics of 116 laboratory-confirmed COVID-19 patients admitted to the East Campus of Renmin Hospital of Wuhan University from January 30, 2020 and March 4, 2020. The East Campus of Renmin Hospital of Wuhan University was one of the medical institutions designated for COVID-19 by the National Health Commission, responsible for the treatment of severe COVID-19 patients in China. In addition, retrospective analysis of clinical data posed no potential risk to the patients. 


\section{Data Collection}

Respiratory tract samples were tested by RT-PCR, and all patients participating in this study showed positive test results. Patients under 18 years of age, pregnant women, and those transferred to other hospitals during hospitalization were excluded. So, a total of 116 patients were included in this study. Patients' demographics, laboratory test results, and the assessment of disease severity at admission were obtained from electronic medical records. Specifically, laboratory parameters included complete blood count (CBC), biochemical parameters, immune function, and coagulation function. According to the SARS-CoV-2 Diagnosis and Treatment Guidelines (6th edition) issued by the National Health Commission, all patients met the clinical diagnostic criteria with the classification of disease severity. Two researchers independently reviewed the data collection forms to verify the accuracy of the data.

\section{Statistical Analysis}

Continuous variables were expressed as mean and standard deviation (SD) or median and interquartile range (IQR), and dichotomous variables were expressed as number of cases and percentage $(n, \%)$. The $t$ test was used for the variables in normal distribution in the two groups of data, the wilcoxon rank sum test was used for non-normal distribution in the two groups of data; the analysis of variance was used for the variables that conform to the normal distribution in the four groups of data, the Kruskal-Wallis $\mathrm{H}$ test was used for the variables that do not conform to the normal distribution in the four groups of data, and Bonferroni correction method was used for pairwise comparison of the variables with different test results; Chi-square test was used for the dichotomous variables, and pairwise comparison was conducted for the variables with different test results. Univariate and multivariate analysis on the factors affecting the time from symptom onset to positive nucleic acid test and the conversion time from positive to negative nucleic acid test. Data were analyzed using $\mathrm{R}$ software, and $p<0.05$ was considered statistically significant difference.

\section{Results}

Association between clinical characteristics and laboratory findings and the time from symptom onset to the first positive nucleic acid test

As shown in Table 1, patients were divided into two groups according to the time from symptom onset to the first positive nucleic acid test. 61 patients were diagnosed with positive nucleic acid within 1 week after onset, who were defined as the non-prolongation group. Of these 61 patients, $23(37.70 \%)$ had severe disease and $38(62.30 \%)$ had mild disease. The remaining 55 patients were diagnosed after more than 7 days and were defined as the prolongation group, in which the proportion of severe patients was $58.18 \%$ (32 patients), and the rate of severe disease in the prolongation group was significantly higher than that in the patients from the non-prolongation group $(p<0.05)$. The demographics and laboratory findings were compared between the two groups. The mean age of the patients in the prolongation group was 61.53 years, higher than that in the non-prolongation group ( 56.46 years), but the difference was not 
statistically significant $(p=0.063)$. The median conversion time from positive to negative nucleic acid test in the prolongation group was 29 days (IQR,16.0-43.5), which was significantly higher than that in the non-prolongation group (23 days, IQR, 15.0-37.75, $p<0.05$ ). In addition, the duration of disease before admission was significantly higher $(p<0.05)$ in the prolongation group. In terms of blood routine examination, the lymphocyte count of patients in the prolongation group was significantly lower than that in the non-prolongation group, however, the comparison of other parameters between these groups showed no statistically significant difference $(p>0.05)$. Similarly, no significant difference of lymphocyte subsets and biochemical parameters $(p>0.05)$ was observed between the two groups. 
Table 1

Comparison of clinical characteristics and laboratory findings between the two groups

Q7 days

Sex, $\mathrm{n}(\%)$

Male

Female

Disease Severity, n (\%)

Mild

Severe

Age (years)

Length of hospital stay (days)

Median conversion time from positive to negative nucleic acid test (days)

Median time from onset of

symptom to hospital admission (days)

White blood cells $\left(\times 10^{9} / \mathrm{L}\right)$

Neutrophils $\left(\times 10^{9} / \mathrm{L}\right)$

Lymphocytes $\left(\times 10^{9} / \mathrm{L}\right)$

Platelets $\left(\times 10^{9} / \mathrm{L}\right)$

$\mathrm{CD} 3+\mathrm{T}$ cell counts (cells/uL)

CD4 $+\mathrm{T}$ cell counts (cells/uL)

$\mathrm{CD} 8+\mathrm{T}$ cell counts (cells/uL)

$\mathrm{CD} 4+/ \mathrm{CD} 8+$ ratio

CD19 + B cell counts (cells/uL)

NK cell counts (cells/uL)

D-dimer $(\mu \mathrm{g} / \mathrm{mL})$

Albumin (g/L)

$0.82(0.40,2.61)$
$8.00(6.00,12.00)$

$42(68.85)$

19 (31.15)

38 (62.30)

$23(37.70)$

$56.46 \pm 13.71$

$36.09 \pm 15.28$

$23.00(15.00,37.75)$

$5.65(4.50,7.81)$

$3.83(2.61,6.43)$

$1.12(0.83,1.55)$

$219.34 \pm 87.23$

$683.00(405.00$ 926.00)

$380.00(237.00$ 626.00)

$229.00(125.00,336.00)$

$1.85(1.23,3.05)$

159.00 (106.00, 250.00)

$96.00(71.00,170.00)$

$35.92 \pm 4.28$

Page 6/24 $\geq 7$ days

$29(52.73)$

0.075

$26(47.27)$

$23(41.82)$

0.027

32 (58.18)

$61.53 \pm 15.42$

0.063

$39.25 \pm 16.01$

0.281

$29.00(16.00,43.50) \quad 0.041$

$13.00(9.50,15.00)<$

0.001

$5.65(4.36,7.11)$

0.477

$3.89(2.84,5.75)$

0.810

$0.94(0.64,1.22)$

0.027

$243.4 \pm 85.61$

0.137

$517(315,821)$

0.091

$346(202,491)$

0.179

$169.00(101.50$

270.50)

0.084

$1.85(1.37,2.82)$

0.636

132.00 (95.00,

0.201 199.00)

92.00 (57.00, 147.00)

0.360

$0.95(0.43,3.68)$

0.713

$35.48 \pm 3.79$

0.561 


\begin{tabular}{|c|c|c|c|}
\hline & Q7 days & $\geq 7$ days & $\begin{array}{l}P \\
\text { value }\end{array}$ \\
\hline Alanine aminotransferase (U/L) & $28.00(16.00,50.00)$ & $\begin{array}{l}27.00(19.00 \\
42.00)\end{array}$ & 0.715 \\
\hline Aspartate aminotransferase $(\mathrm{U} / \mathrm{L})$ & $30.00(21.00,48.00)$ & $\begin{array}{l}30.00(19.00 \\
40.00)\end{array}$ & 0.281 \\
\hline Alkaline phosphatase $(\mathrm{U} / \mathrm{L})$ & $60.00(51.00,79.00)$ & $\begin{array}{l}64.00(50.00 \\
92.00)\end{array}$ & 0.521 \\
\hline Y-glutamyl transpeptidase (U/L) & $34.00(19.00,69.00)$ & $\begin{array}{l}30.00(21.00 \\
56.00)\end{array}$ & 0.678 \\
\hline Total bilirubin $(\mu \mathrm{mol} / \mathrm{L})$ & $10.70(8.90,13.80)$ & $10.70(7.70,15.75)$ & 0.923 \\
\hline Serum creatinine $(\mu \mathrm{mol} / \mathrm{L})$ & $61.00(50.00,72.00)$ & $\begin{array}{l}63.00(50.00 \\
71.00)\end{array}$ & 0.894 \\
\hline Lactate dehydrogenase (U/L) & $\begin{array}{l}266.00 \text { (203.00, } \\
391.00)\end{array}$ & $\begin{array}{l}298.00(229.00 \\
370.00)\end{array}$ & 0.682 \\
\hline C-reactive protein (mg/L) & $37.50(17.20,73.60)$ & $\begin{array}{l}37.50(11.95 \\
59.65)\end{array}$ & 0.463 \\
\hline
\end{tabular}

As shown in Table 2, univariate Cox regression analysis indicated that the prolonged time from positive to negative nucleic acid test was a risk factor for the time from symptom onset to positive nucleic acid test $(p=0.018)$, while disease severity $(p=0.044)$, disease duration before admission $(p<0.001)$, age $(p=$ $0.04)$, and platelet count $(p=0.035)$ were protective factors for the time from symptom onset to positive nucleic acid test. In order to systematically analyze the factors affecting the length of time from symptom onset to first positive nucleic acid in patients, disease severity, disease duration before admission, age and platelet (PLT) were included in the multivariate Cox regression model as independent variables based on the analysis results of univariate Cox regression and our expertise on COVID-19. Multivariate analysis showed that disease severity ( $\mathrm{HR}=0.572 ; 95 \% \mathrm{Cl} 0.348-0.942 ; p=0.028)$ and disease duration before admission ( $\mathrm{HR}=0.853 ; 95 \% \mathrm{Cl} 0.805-0.904 ; p<0.001)$ were protective factors for the time from symptom onset to positive nucleic acid test, and the time from positive to negative nucleic acid test $(\mathrm{HR}=1.030 ; 95 \% \mathrm{Cl} 1.020-1.040 ; p<0.001)$ was a risk factor for the time from symptom onset to positive nucleic acid test. 
Table 2

Univariate and multivariate Cox regression model analysis results of the time from symptom onset to positive nucleic acid test

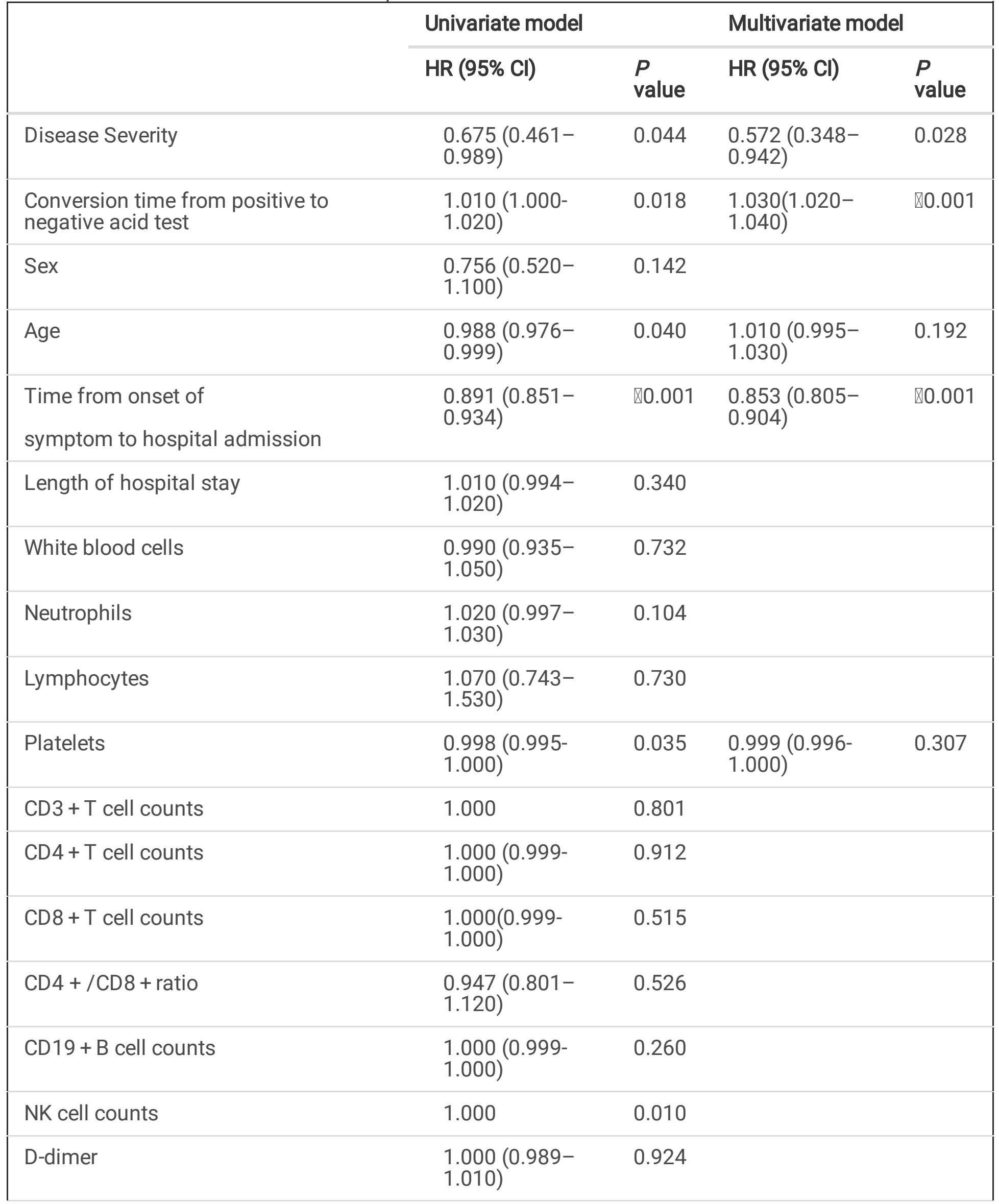




\begin{tabular}{|c|c|c|c|c|}
\hline & \multicolumn{2}{|l|}{ Univariate model } & \multicolumn{2}{|c|}{ Multivariate model } \\
\hline & $\mathrm{HR}(95 \% \mathrm{Cl})$ & $\begin{array}{l}P \\
\text { value }\end{array}$ & $\mathrm{HR}(95 \% \mathrm{Cl})$ & $\begin{array}{l}P \\
\text { value }\end{array}$ \\
\hline Alanine aminotransferase & $\begin{array}{l}1.000(0.997- \\
1.010)\end{array}$ & 0.416 & & \\
\hline Aspartate aminotransferase & $\begin{array}{l}1.010(0.998- \\
1.020)\end{array}$ & 0.142 & & \\
\hline Alkaline phosphatase & $\begin{array}{l}0.996(0.990- \\
1.000)\end{array}$ & 0.245 & & \\
\hline Y-glutamyl transpeptidase & $\begin{array}{l}1.000(0.998- \\
1.000)\end{array}$ & 0.467 & & \\
\hline Albumin & $\begin{array}{l}1.010(0.965- \\
1.060)\end{array}$ & 0.621 & & \\
\hline Total bilirubin & $\begin{array}{l}0.985(0.955- \\
1.020)\end{array}$ & 0.329 & & \\
\hline Serum creatinine & $\begin{array}{l}1.000(0.994- \\
1.01)\end{array}$ & 0.616 & & \\
\hline Lactate dehydrogenase & $\begin{array}{l}1.000(0.999- \\
1.000)\end{array}$ & 0.905 & & \\
\hline C-reactive protein & $\begin{array}{l}0.997(0.994- \\
1.000)\end{array}$ & 0.097 & & \\
\hline
\end{tabular}

\section{Association between clinical characteristics and laboratory findings and the length of negative conversion time of SARS-COV-2 virus in patients}

As shown in Table 3, patients were divided into four groups according to the length of conversion time from the first positive to negative nucleic acid test. Of all the patients in these groups, 23 experienced less than 2 weeks of this conversion time, 40 experienced 2-4 weeks, 26 experienced 4- 6 weeks, and the remaining 27 experienced more than 6 weeks. The basic characteristics of patients and laboratory findings were compared among these groups which showed that the most common symptom on admission was fever $(75.86 \%)$, followed by cough $(62.93 \%)$, fatigue $(21.55 \%)$, Shortness of breath $(21.55 \%)$, and diarrhea (10.34\%) dyspnea (9.48\%). The most common comorbidity was hypertension (25.86\%), followed by diabetes (16.38\%), and cardiovascular disease (12.07\%), however, no significant differences were found these symptoms and comorbidities among these groups $(p>0.05)$. 
Table 3

Comparison of clinical characteristics and laboratory findings among the four groups

\begin{tabular}{|c|c|c|c|c|c|}
\hline & 凶2 weeks & $2 \bigotimes 4$ weeks & $\begin{array}{l}\text { 4『6 } \\
\text { weeks }\end{array}$ & $\begin{array}{l}\geq 6 \\
\text { weeks }\end{array}$ & $\begin{array}{l}P \\
\text { value }\end{array}$ \\
\hline Sex, n (\%) & & & & & 0.880 \\
\hline Male & $12(52.17)$ & $23(57.50)$ & $13(50.00)$ & $\begin{array}{l}13 \\
(48.15)\end{array}$ & \\
\hline Female & $11(47.83)$ & $17(42.50)$ & $13(50.00)$ & $\begin{array}{l}14 \\
(51.85)\end{array}$ & \\
\hline Disease Severity, n (\%) & & & & & 0.709 \\
\hline Mild & $13(56.52)$ & $26(65.00)$ & $\begin{array}{l}14 \\
(53.85)\end{array}$ & $\begin{array}{l}18 \\
(66.67)\end{array}$ & \\
\hline Severe & $10(43.48)$ & $14(35.00)$ & $\begin{array}{l}12 \\
(46.15)\end{array}$ & $9(33.33)$ & \\
\hline Age (years) & $\begin{array}{l}64.74 \pm \\
14.32\end{array}$ & $56.15 \pm 14.58$ & $\begin{array}{l}60.73 \pm \\
14.8\end{array}$ & $\begin{array}{l}56.07 \pm \\
14.1\end{array}$ & 0.091 \\
\hline \multicolumn{6}{|l|}{ Symptoms and signs, n (\%) } \\
\hline Fever & $17(73.91)$ & $29(72.5)$ & $\begin{array}{l}19 \\
(73.08)\end{array}$ & $\begin{array}{l}23 \\
(85.19)\end{array}$ & 0.640 \\
\hline Cough & $15(65.22)$ & $28(70.00)$ & $\begin{array}{l}12 \\
(46.15)\end{array}$ & $\begin{array}{l}18 \\
(66.67)\end{array}$ & 0.240 \\
\hline Shortness of breath & $8(34.78)$ & $6(15.00)$ & $8(30.77)$ & $3(11.11)$ & 0.092 \\
\hline Dyspnea & $2(8.70)$ & $2(5.00)$ & $3(11.54)$ & $4(14.81)$ & 0.578 \\
\hline Fatigue & $8(34.78)$ & $5(12.50)$ & $7(26.92)$ & $5(18.52)$ & 0.178 \\
\hline Diarrhea & $3(13.04)$ & $4(10.00)$ & $3(11.54)$ & $2(7.41)$ & 0.924 \\
\hline \multicolumn{6}{|l|}{ Comorbidities, n (\%) } \\
\hline Hypertension & $8(34.78)$ & $6(15.00)$ & $7(26.92)$ & $9(33.33)$ & 0.239 \\
\hline Diabetes & $5(21.74)$ & $7(17.50)$ & $4(15.38)$ & $3(11.11)$ & 0.781 \\
\hline $\begin{array}{l}\text { Cardiovascular and } \\
\text { cerebrovascular disorders }\end{array}$ & $4(17.39)$ & $2(5.00)$ & $4(15.38)$ & $4(14.81)$ & 0.398 \\
\hline \multicolumn{6}{|c|}{ a indicates $p<0.05$ in the pairwise comparison with the $<2$ weeks group; } \\
\hline \multicolumn{6}{|c|}{$\mathrm{b}$ indicates $p<0.05$ when compared with the 2 to 4 weeks group; } \\
\hline \multicolumn{6}{|c|}{${ }^{\mathrm{C}}$ indicates $p<0.05$ when compared with the 4 to 6 weeks group; } \\
\hline
\end{tabular}




\begin{tabular}{|c|c|c|c|c|c|}
\hline & \2 weeks & 2ه4 weeks & $\begin{array}{l}4 \llbracket 6 \\
\text { weeks }\end{array}$ & $\begin{array}{l}\geq 6 \\
\text { weeks }\end{array}$ & $\begin{array}{l}P \\
\text { value }\end{array}$ \\
\hline Other comorbidities & $10(43.48)$ & $19(47.50)$ & $\begin{array}{l}10 \\
(38.46)\end{array}$ & $7(25.93)$ & 0.346 \\
\hline $\begin{array}{l}\text { Median time from symptom } \\
\text { onset to positive nucleic acid } \\
\text { test (days) }\end{array}$ & $4(0,8)$ & $5(2.5,8.25)$ & $\begin{array}{l}6.5(3.5 \\
10)\end{array}$ & $\begin{array}{l}9(6 \\
14.5)^{\mathrm{a}}\end{array}$ & 0.007 \\
\hline $\begin{array}{l}\text { Median time from onset of } \\
\text { symptom to hospital admission } \\
\text { (days) }\end{array}$ & $7(5.5,13)$ & $10(7,10.5)$ & $\begin{array}{l}12(8.25 \\
14.75)\end{array}$ & $\begin{array}{l}12(8.5 \\
14.5)^{b}\end{array}$ & 0.020 \\
\hline White blood cells $\left(\times 10^{9} / \mathrm{L}\right)$ & $\begin{array}{l}5.51(4.37, \\
7.70)\end{array}$ & $\begin{array}{l}5.37(4.42, \\
6.74)\end{array}$ & $\begin{array}{l}6.23 \\
(4.61 \\
10.06)\end{array}$ & $\begin{array}{l}5.65 \\
(4.49 \\
7.12)\end{array}$ & 0.507 \\
\hline Neutrophils $\left(\times 10^{9} / \mathrm{L}\right)$ & $\begin{array}{l}3.89(2.98, \\
5.86)\end{array}$ & $\begin{array}{l}3.46(2.74, \\
5.08)\end{array}$ & $\begin{array}{l}4.38 \\
(2.93 \\
9.16)\end{array}$ & $\begin{array}{l}3.83 \\
(2.44 \\
5.83)\end{array}$ & 0.481 \\
\hline Lymphocytes $\left(\times 10^{9} / \mathrm{L}\right)$ & $1.05 \pm 0.44$ & $1.14 \pm 0.61$ & $\begin{array}{l}1.06 \pm \\
0.46\end{array}$ & $\begin{array}{l}1.16 \pm \\
0.58\end{array}$ & 0.816 \\
\hline Platelets $\left(\times 10^{9} / \mathrm{L}\right)$ & $\begin{array}{l}231.70 \pm \\
74.90\end{array}$ & $\begin{array}{l}229.38 \pm \\
100.81\end{array}$ & $\begin{array}{l}220.92 \pm \\
76.62\end{array}$ & $\begin{array}{l}241.44 \pm \\
87.01\end{array}$ & 0.864 \\
\hline $\mathrm{CD} 3+\mathrm{T}$ cell counts (cells/uL) & $\begin{array}{l}510.00 \\
(323.50 \\
854.50)\end{array}$ & $\begin{array}{l}602.50(358.70 \\
927.20)\end{array}$ & $\begin{array}{l}631.00 \\
(342.50 \\
830.00)\end{array}$ & $\begin{array}{l}670.00 \\
(435.00 \\
926.50)\end{array}$ & 0.818 \\
\hline CD $4+T$ cell counts (cells/uL) & $\begin{array}{l}381.00 \\
(217.50 \\
495.00)\end{array}$ & $\begin{array}{l}352.50(221.00 \\
551.00)\end{array}$ & $\begin{array}{l}377.00 \\
(220.00 \\
562.00)\end{array}$ & $\begin{array}{l}443.00 \\
(216.00 \\
528.00)\end{array}$ & 0.960 \\
\hline CD8 $+\mathrm{T}$ cell counts (cells/uL) & $\begin{array}{l}118.00 \\
(91.50 \\
261.00)\end{array}$ & $\begin{array}{l}249.00(129.75 \\
337.50)\end{array}$ & $\begin{array}{l}194.00 \\
(76.00 \\
305.50)\end{array}$ & $\begin{array}{l}207.00 \\
(134.00 \\
319.00)\end{array}$ & 0.151 \\
\hline CD4 + /CD8 + ratio & $\begin{array}{l}2.53(1.62, \\
3.40)\end{array}$ & $\begin{array}{l}1.60(1.07 \\
2.16)^{\mathrm{a}}\end{array}$ & $\begin{array}{l}2.44 \\
(1.26 \\
3.23)\end{array}$ & $\begin{array}{l}1.85 \\
(1.20 \\
3.09)\end{array}$ & 0.037 \\
\hline CD19 + B cell counts (cells/uL) & $\begin{array}{l}143.00 \\
(113.00 \\
212.50)\end{array}$ & $\begin{array}{l}118.00(87.00 \\
177.25)\end{array}$ & $\begin{array}{l}156.00 \\
(86.00 \\
245.25)\end{array}$ & $\begin{array}{l}159.00 \\
(104.50 \\
273.50)\end{array}$ & 0.495 \\
\hline
\end{tabular}

a indicates $p<0.05$ in the pairwise comparison with the $<2$ weeks group;

$\mathrm{b}$ indicates $p<0.05$ when compared with the 2 to 4 weeks group;

${ }^{c}$ indicates $p<0.05$ when compared with the 4 to 6 weeks group;

d indicates $p<0.05$ when compared with the $\geq 6$ weeks group. 


\begin{tabular}{|c|c|c|c|c|c|}
\hline & \2 weeks & $2 ه 4$ weeks & 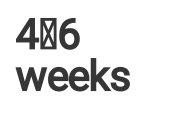 & $\begin{array}{l}\geq 6 \\
\text { weeks }\end{array}$ & $\begin{array}{l}P \\
\text { value }\end{array}$ \\
\hline NK cell counts (cells/uL) & $\begin{array}{l}95.00 \\
(66.50 \\
132.00)\end{array}$ & $\begin{array}{l}105.00(72.50 \\
174.75)\end{array}$ & $\begin{array}{l}59.00 \\
(40.25 \\
125.00)\end{array}$ & $\begin{array}{l}109.00 \\
(78.50 \\
172.50)\end{array}$ & 0.074 \\
\hline D-dimer $(\mu \mathrm{g} / \mathrm{mL})$ & $\begin{array}{l}1.05(0.62 \\
4.51)\end{array}$ & $\begin{array}{l}0.70(0.36 \\
2.42)\end{array}$ & $\begin{array}{l}0.84 \\
(0.36 \\
3.21)\end{array}$ & $\begin{array}{l}0.82 \\
(0.48 \\
2.70)\end{array}$ & 0.370 \\
\hline Alanine aminotransferase $(\mathrm{U} / \mathrm{L})$ & $\begin{array}{l}27.00 \\
(20.50 \\
42.50)\end{array}$ & $\begin{array}{l}29.00(16.75 \\
42.00)\end{array}$ & $\begin{array}{l}24.50 \\
(16.75 \\
50.75)\end{array}$ & $\begin{array}{l}27.00 \\
(18.00 \\
44.00)\end{array}$ & 0.966 \\
\hline $\begin{array}{l}\text { Aspartate aminotransferase } \\
(\mathrm{U} / \mathrm{L})\end{array}$ & $\begin{array}{l}30.00 \\
(20.00 \\
45.00)\end{array}$ & $\begin{array}{l}29.50(20.75 \\
39.25)\end{array}$ & $\begin{array}{l}31.50 \\
(19.25 \\
43.00)\end{array}$ & $\begin{array}{l}34.00 \\
(19.50 \\
45.00)\end{array}$ & 0.938 \\
\hline Alkaline phosphatase (U/L) & $\begin{array}{l}77.00 \\
(57.00 \\
90.50)\end{array}$ & $\begin{array}{l}59.00(48.75 \\
84.50)\end{array}$ & $\begin{array}{l}59.00 \\
(50.50 \\
72.75)\end{array}$ & $\begin{array}{l}65.00 \\
(51.00 \\
86.50)\end{array}$ & 0.264 \\
\hline Y-glutamyl transpeptidase $(\mathrm{U} / \mathrm{L})$ & $\begin{array}{l}40.00 \\
(20.00 \\
57.00)\end{array}$ & $\begin{array}{l}30.50(21.00 \\
68.25)\end{array}$ & $\begin{array}{l}31.00 \\
(20.25 \\
38.00)\end{array}$ & $\begin{array}{l}31.00 \\
(20.00 \\
57.00)\end{array}$ & 0.925 \\
\hline Total bilirubin $(\mu \mathrm{mol} / \mathrm{L})$ & $\begin{array}{l}11.30 \\
(8.05, \\
16.05)\end{array}$ & $\begin{array}{l}10.50(7.83 \\
16.43)\end{array}$ & $\begin{array}{l}10.90 \\
(8.32 \\
13.57)\end{array}$ & $\begin{array}{l}10.30 \\
(8.80, \\
14.00)\end{array}$ & 0.960 \\
\hline Serum creatinine $(\mu \mathrm{mol} / \mathrm{L})$ & $\begin{array}{l}65.00 \\
(55.50 \\
77.50)\end{array}$ & $\begin{array}{l}63.50(49.17 \\
70.00)\end{array}$ & $\begin{array}{l}56.50 \\
(47.25 \\
69.50)\end{array}$ & $\begin{array}{l}59.00 \\
(49.00 \\
69.00)\end{array}$ & 0.571 \\
\hline Lactate dehydrogenase (U/L) & $\begin{array}{l}300.00 \\
(230.50 \\
381.50)\end{array}$ & $\begin{array}{l}261.50 \\
(206.70,328.20)\end{array}$ & $\begin{array}{l}307.50 \\
(244.00 \\
378.00)\end{array}$ & $\begin{array}{l}242.00 \\
(210.50 \\
442.00)\end{array}$ & 0.454 \\
\hline C-reactive protein (mg/L) & $\begin{array}{l}50.90 \\
(28.70 \\
92.45)\end{array}$ & $\begin{array}{l}37.5(17.18 \\
62.73)\end{array}$ & $\begin{array}{l}37.50 \\
(5.78, \\
63.09)\end{array}$ & $\begin{array}{l}37.5 \\
(17.00 \\
48.10)\end{array}$ & 0.213 \\
\hline \multicolumn{6}{|c|}{ a indicates $p<0.05$ in the pairwise comparison with the $<2$ weeks group; } \\
\hline \multicolumn{6}{|c|}{$\mathrm{b}$ indicates $p<0.05$ when compared with the 2 to 4 weeks group; } \\
\hline \multicolumn{6}{|c|}{${ }^{c}$ indicates $p<0.05$ when compared with the 4 to 6 weeks group; } \\
\hline es $n<0,05$ when com & th th & our & & & \\
\hline
\end{tabular}

The median time from symptom onset to positive nucleic acid test was 9 days (IQR, 6.0-14.5) in patients with the conversion time of more than 6 weeks from positive to negative nucleic acid test, which was significantly longer than that in patients with the conversion time of less than 2 weeks (4 days, IQR, $0-$ 8.0) $(p<0.05)$. In addition, patients with the conversion time of more than 6 weeks, had longer disease 
duration before admission, compared with patients with the conversion time of 2 to 4 weeks $(p<0.05)$. In terms of the blood routine examination, there were no statistically significant differences in white blood cell count, neutrophil count and lymphocyte count among these groups $(p>0.05)$. As for immunological parameters, the CD4/CD8 ratio was lower in patients with conversion time of 2 to 4 weeks than in patients with conversion time of less than 2 weeks $(p<0.05)$. However, CD $3+T$ cell, CD $4+T$ cell, and NK cell counts did not show statistically significant differences between these groups $(p>0.05)$.

As shown in Table 4, univariate Cox regression analysis indicated that the time from symptom onset to positive nucleic acid test $(p=0.019)$, disease duration before admission $(p=0.004)$, length of hospital stay $(p<0.001)$, and duration of virus shedding $(p<0.001)$ were significantly correlated with the negative conversion time of SARS-CoV-2 virus. Combined with the results of univariate Cox regression analysis and our expertise in COVID-19, the time from symptom onset to positive nucleic acid test, duration disease before admission, length of hospital stay, duration of virus shedding, and age were included as independent variables in the multivariate Cox regression model, and the results showed that the time from symptom onset to positive nucleic acid test $(\mathrm{HR}=1.010 ; 95 \% \mathrm{Cl} 1.005-1.020 ; p=0.0282)$ was an independent risk factor for prolonged negative conversion from positive to negative nucleic acid test. 
Table 4

Univariate and multivariate cox regression model analysis results of the conversion time from positive to negative nucleic acid test

\begin{tabular}{|c|c|c|c|c|}
\hline & \multicolumn{2}{|c|}{ Univariate model } & \multicolumn{2}{|c|}{ Multivariate model } \\
\hline & $\mathrm{HR}(95 \% \mathrm{Cl})$ & $\begin{array}{l}P \\
\text { value }\end{array}$ & $\mathrm{HR}(95 \% \mathrm{Cl})$ & $\begin{array}{l}P \\
\text { value }\end{array}$ \\
\hline Disease severity & $\begin{array}{l}1.120(0.771- \\
1.640)\end{array}$ & 0.544 & & \\
\hline $\begin{array}{l}\text { Time from symptom onset to positive } \\
\text { nucleic acid test }\end{array}$ & $\begin{array}{l}1.040(1.010- \\
1.080)\end{array}$ & 0.019 & $\begin{array}{l}1.010(1.005- \\
1.020)\end{array}$ & 0.028 \\
\hline Sex & $\begin{array}{l}0.769(0.528- \\
1.120)\end{array}$ & 0.172 & & \\
\hline Fever & $\begin{array}{l}0.725(0.471- \\
1.120)\end{array}$ & 0.145 & & \\
\hline Cough & $\begin{array}{l}0.857(0.582- \\
1.260)\end{array}$ & 0.436 & & \\
\hline Shortness of breath & $\begin{array}{l}1.310(0.836- \\
2.040)\end{array}$ & 0.241 & & \\
\hline Dyspnea & $\begin{array}{l}0.754(0.403- \\
1.41)\end{array}$ & 0.376 & & \\
\hline Fatigue & $\begin{array}{l}1.090(0.700- \\
1.700)\end{array}$ & 0.698 & & \\
\hline Diarrhea & $\begin{array}{l}1.080(0.586- \\
1.980)\end{array}$ & 0.813 & & \\
\hline Hypertension & $\begin{array}{l}1.000(0.659- \\
1.530)\end{array}$ & 0.990 & & \\
\hline Diabetes & $\begin{array}{l}1.510(0.922- \\
2.490)\end{array}$ & 0.101 & & \\
\hline Other comorbidities & $\begin{array}{l}1.140(0.774- \\
1.670)\end{array}$ & 0.514 & & \\
\hline Age & $\begin{array}{l}1.010(0.996- \\
1.020)\end{array}$ & 0.179 & $\begin{array}{l}1.000(0.984- \\
1.020)\end{array}$ & 0.192 \\
\hline $\begin{array}{l}\text { Time from onset of } \\
\text { symptom to hospital admission }\end{array}$ & $\begin{array}{l}0.937(0.896- \\
0.980)\end{array}$ & 0.004 & $\begin{array}{l}1.000(0.944- \\
1.060)\end{array}$ & 0.602 \\
\hline Length of hospital stay & $\begin{array}{l}0.921(0.905- \\
0.938)\end{array}$ & $\varangle 0.001$ & $\begin{array}{l}1.000(0.979- \\
1.020)\end{array}$ & 0.669 \\
\hline White blood cells & $\begin{array}{l}1.01(0.949- \\
1.070)\end{array}$ & 0.808 & & \\
\hline
\end{tabular}




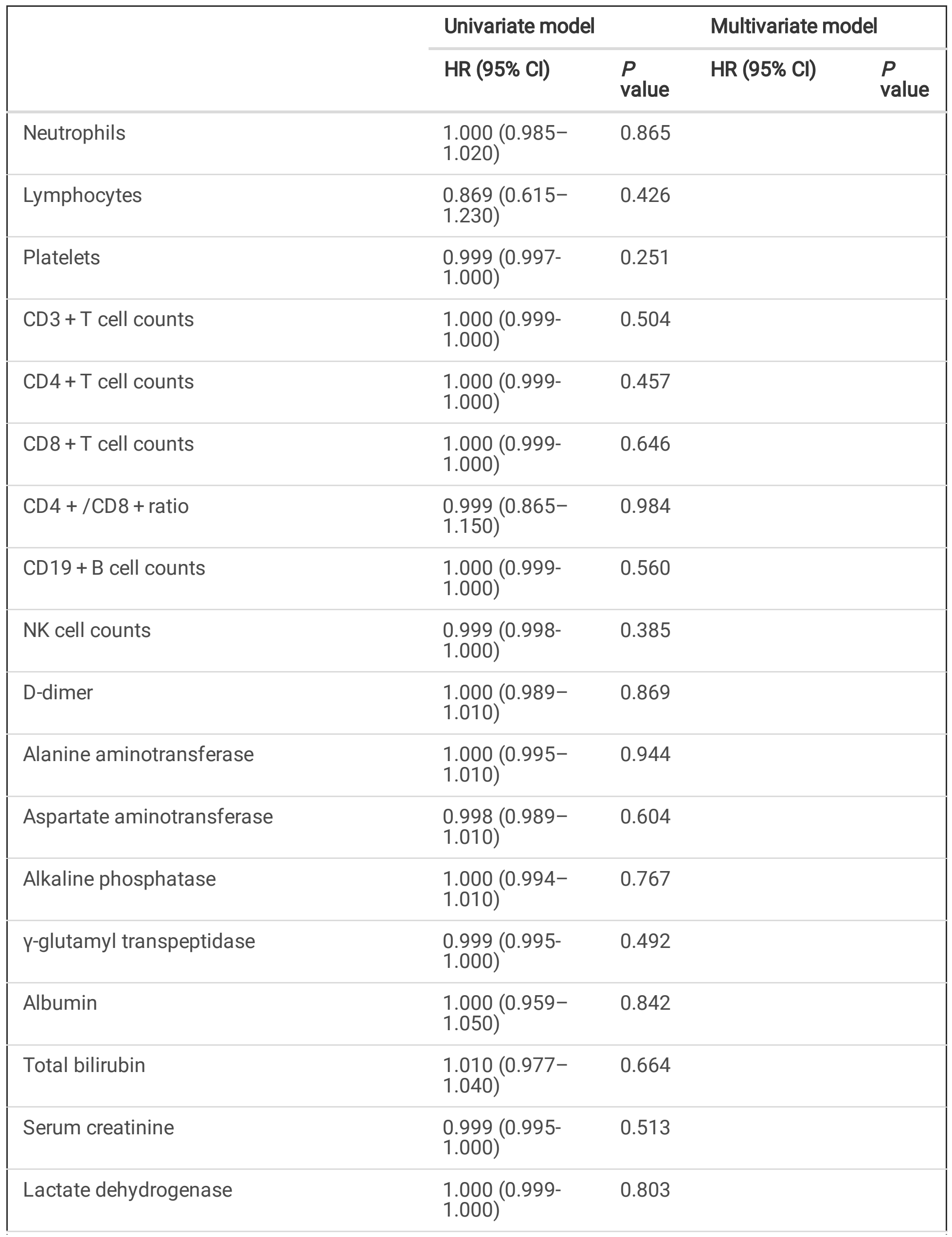




\begin{tabular}{|lllll|}
\hline & Univariate model & \multicolumn{2}{l|}{ Multivariate model } \\
\cline { 2 - 4 } & HR $(95 \% \mathrm{Cl})$ & $\begin{array}{l}\boldsymbol{P} \\
\text { value }\end{array}$ & $\mathrm{HR}(95 \% \mathrm{Cl})$ & $\begin{array}{c}\boldsymbol{P} \\
\text { value }\end{array}$ \\
\hline C-reactive protein & $1.000(1.000-$ & 0.078 & \\
\hline
\end{tabular}

\section{Association between clinical characteristics and laboratory findings and disease severity in patients}

As shown in Table 5, the relevant parameters between mild and severe patients were compared. The mean age of severe patients was 70.49 years, which was significantly higher than that of mild patients (51.49 years, $p<0.05$ ). Among the mild patients, $50.70 \%$ were male, slightly lower than that in severe patients (55.56\%), but the difference between the two groups was not statistically significant. The median time from symptom onset to the first positive nucleic acid test was 5.0 days (IQR, 2.5-8.0) in mild patients, significantly shorter than 8 days (IQR, 4.0-12.0) in severe patients $(p=0.047)$. The median conversion time from the first positive to negative nucleic acid test was 24 days (IQR, 15.5-41.5) and 25 days (14.0-38.0) in mild and severe patients, respectively $(p=0.671)$. In terms of the blood routine examination, the white blood cell count and neutrophil count were increased to varying degrees, and the lymphocyte count was decreased $(p<0.05)$ in severe patients than in mild patients. As for the immunological indicators, CD $3+T$ cells and CD $8+T$ cells were significantly lower in severe patients $(p<$ $0.05)$, compared with those in mild patients, and there were no statistically significant differences in CD4 $+T$ cells and NK cell counts between the two groups $(p>0.05)$. In terms of the biochemical parameters, albumin concentration was significantly higher in mild patients than that in severe patients $(p<0.05)$. Alanine aminotransferase (ALT), aspartate aminotransferase (AST), alkaline phosphatase (ALP) and $\gamma^{-}$ glutamyl transpeptidase ( $\mathrm{y}-\mathrm{GGT}$ ) in severe patients were slightly higher than those in mild patients, but the difference was not statistically significant between the two groups $(p>0.05)$. 
Table 5

Comparison of clinical characteristics and laboratory findings between mild and severe patients with COVID-19

\begin{tabular}{|c|c|c|c|}
\hline & Mild & Severe & $\begin{array}{l}P \\
\text { value }\end{array}$ \\
\hline \multicolumn{4}{|l|}{ Sex, n (\%) } \\
\hline Male & $36(50.70)$ & $25(55.56)$ & 0.610 \\
\hline Female & $35(49.30)$ & $20(44.44)$ & \\
\hline Age (years) & $51.49 \pm 12.30$ & $70.49 \pm 9.90$ & $\varangle 0.001$ \\
\hline Length of hospital stay (days) & $\begin{array}{l}37.00(23.50 \\
46.00)\end{array}$ & $40(31,47)$ & 0.309 \\
\hline $\begin{array}{l}\text { Median time from symptom onset to positive } \\
\text { nucleic acid test (days) }\end{array}$ & $5(2.5,8)$ & $8(4,12)$ & 0.047 \\
\hline $\begin{array}{l}\text { Median conversion time from positive to negative } \\
\text { nucleic acid test (days) }\end{array}$ & $24(15.5,41.5)$ & $25(14,38)$ & 0.671 \\
\hline $\begin{array}{l}\text { Median time from onset of } \\
\text { symptom to hospital admission (days) }\end{array}$ & $10(7,14)$ & $10(7,14)$ & 0.776 \\
\hline White blood cells $\left(\times 10^{9} / \mathrm{L}\right)$ & $5.48(4.13,6.82)$ & $6.49(4.75,9.00)$ & 0.026 \\
\hline Neutrophils $\left(\times 10^{9} / \mathrm{L}\right)$ & $3.45(2.43,4.70)$ & $4.88(3.26,7.47)$ & 0.002 \\
\hline Lymphocytes $\left(\times 10^{9} / \mathrm{L}\right)$ & $1.14(0.80,1.55)$ & $0.93(0.61,1.13)$ & 0.009 \\
\hline Platelets $\left(\times 10^{9} / \mathrm{L}\right)$ & $235.27 \pm 89.86$ & $223.62 \pm 82.58$ & 0.484 \\
\hline CD3 $+\mathrm{T}$ cell counts (cells/uL) & $\begin{array}{l}684.00(431.50 \\
958.50)\end{array}$ & $\begin{array}{l}503.00(306.00 \\
723.00)\end{array}$ & 0.011 \\
\hline $\mathrm{CD} 4+\mathrm{T}$ cell counts (cells/uL) & $\begin{array}{l}394.00(223.50 \\
613.50)\end{array}$ & $\begin{array}{l}333.00(197.00 \\
476.00)\end{array}$ & 0.119 \\
\hline CD8 $+T$ cell counts (cells/uL) & $\begin{array}{l}259.00(130.50 \\
354.00)\end{array}$ & $\begin{array}{l}132.00(96.00 \\
222.00)\end{array}$ & 0.001 \\
\hline CD4 + /CD8 + ratio & $1.66(1.15,2.66)$ & $2.16(1.47,3.33)$ & 0.020 \\
\hline CD19 + B cell counts (cells/uL) & $\begin{array}{l}158.00(106.00 \\
248.50)\end{array}$ & $\begin{array}{l}137.00(85.00 \\
193.00)\end{array}$ & 0.150 \\
\hline NK cell counts (cells/uL) & $\begin{array}{l}98.00(69.50 \\
180.50)\end{array}$ & $\begin{array}{l}92.00(49.00 \\
136.00)\end{array}$ & 0.182 \\
\hline D-dimer $(\mu \mathrm{g} / \mathrm{mL})$ & $0.59(0.34,1.42)$ & $1.63(0.71,5.36)$ & $\varangle 0.001$ \\
\hline Albumin (g/L) & $37.14 \pm 3.97$ & $33.47 \pm 3.04$ & $₫ 0.001$ \\
\hline
\end{tabular}




\begin{tabular}{|c|c|c|c|}
\hline & Mild & Severe & $\begin{array}{l}P \\
\text { value }\end{array}$ \\
\hline Alanine aminotransferase (U/L) & $\begin{array}{l}27.00(1800 \\
45.50)\end{array}$ & $\begin{array}{l}28.00(18.00 \\
45.00)\end{array}$ & 0.823 \\
\hline Aspartate aminotransferase $(\mathrm{U} / \mathrm{L})$ & $\begin{array}{l}30.00(20.00 \\
40.00)\end{array}$ & $\begin{array}{l}31.00(20.00 \\
44.00)\end{array}$ & 0.509 \\
\hline Alkaline phosphatase $(\mathrm{U} / \mathrm{L})$ & $\begin{array}{l}59.00(49.00 \\
78.50)\end{array}$ & $\begin{array}{l}67.00(52.00 \\
92.00)\end{array}$ & 0.106 \\
\hline Y-glutamyl transpeptidase (U/L) & $\begin{array}{l}31.00(19.50 \\
60.50)\end{array}$ & $\begin{array}{l}32.00(21.00 \\
68.00)\end{array}$ & 0.465 \\
\hline Total bilirubin $(\mu \mathrm{mol} / \mathrm{L})$ & $\begin{array}{l}9.90(7.60 \\
12.55)\end{array}$ & $\begin{array}{l}13.10(9.50 \\
18.30)\end{array}$ & 0.003 \\
\hline Serum creatinine $(\mu \mathrm{mol} / \mathrm{L})$ & $\begin{array}{l}58.00(46.50 \\
68.00)\end{array}$ & $\begin{array}{l}67.00(57.00 \\
75.00)\end{array}$ & 0.005 \\
\hline Lactate dehydrogenase (U/L) & $\begin{array}{l}251.00(208.50 \\
325.00)\end{array}$ & $\begin{array}{l}324.00(242.00 \\
404.00)\end{array}$ & 0.019 \\
\hline C-reactive protein (mg/L) & $\begin{array}{l}37.50(13.05 \\
56.70)\end{array}$ & $\begin{array}{l}41.80(17.20 \\
89.50)\end{array}$ & 0.061 \\
\hline
\end{tabular}

\section{Discussion}

In this study, we retrospectively analyzed the clinical characteristics of 116 laboratory-confirmed COVID19 patients, and explored the factors associated with the length of time from symptom onset to the first positive nucleic acid test of throat swabs, as well as the risk factors for prolonged conversion time from positive to negative nucleic acid test. We concluded that the prolonged time from symptom onset to positive nucleic acid detection was an independent risk factor for prolonged negative conversion time of SARS-CoV-2. Meanwhile, patients with more severe disease and longer disease duration had a shorter time from symptom onset to positive nucleic acid test; on the contrary, patients with longer conversion time from positive to negative nucleic acid test, showed a longer time from symptom onset to positive nucleic acid test.

The strategy of comprehensive and scientific detection of SARS-COV-2 in specific populations and environments through PCR method provides support for the COVID-19 pandemic prevention and control in China $(8,9)$. Nucleic acid screening helps identify infected individuals in a timely manner and prevents the spread of this pandemic (10). Nucleic acid detection of SARS-COV-2 virus by RT-PCR is considered the gold standard for COVID-19 diagnosis (2). At present, testing of throat swabs samples is mainly used for the diagnosis of suspected cases, population screening and staff health monitoring, but the results may be interfered by the patient's viral load, the quality of samples, the mutation and recombination of SARSCoV-2 and other factors, leading to false negative results $(7,11)$. Younger patients are more likely to have false negative results in the early stages of the disease (12). Therefore, the New Coronavirus Pneumonia 
Prevention and Control Protocol (6th edition) states that negative nucleic acid test result does not rule out SARS-COV-2 virus infection. Several studies have reported that RT-PCR produces false negative results, including one study from Beijing which reported a case with two consecutive false negative RT-PCR results (13). Our study found that patients who were tested positive for nucleic acid within a week from the onset of symptoms had a significantly lower rate of severe disease than those who were tested more than a week later. Therefore, there is a need to develop highly sensitive and specific test methods, to improve RT-PCR assays and serological analysis (14), in order to reduce false negative results and promote the timely diagnosis, and eventually to reduce the rate of severe patients. At the same time, our study found that disease severity was a protective factor for the time from symptom onset to positive nucleic acid test: the more severe the patients' disease, the shorter the time from symptom onset to positive nucleic acid test. The reason may be related to the significantly higher viral load in severe patients than in mild patients (15). Studies have reported that the viral load of nasopharyngeal swabs in severe patients can even reach 60 times that of mild patients (16). However, a retrospective study from New York University found that the initial viral load was significantly higher in mild COVID-19 patients than in those severe patients who required hospitalization (17). Further studies are needed to further investigate the relationship between disease severity and viral load in COVID-19 patients.

The COVID-19 prevention and control protocol was based on the detection of SARS-CoV-2 nucleic acid in samples of respiratory tract or blood by real-time fluorescent RT-PCR to determine diagnostic and discharge criteria for patients (18). However, there is no consistent standard to accurately define the duration of SARS-CoV-2 virus infection in the diagnosis and treatment of this disease in actual clinical environment. Only a few studies have analyzed the factors influencing the conversion time from positive to negative test using throat swabs samples for nucleic acid detection. Our study found that the prolonged time from symptom onset to positive nucleic acid test was an independent risk factor for prolonged negative conversion time of SARS-CoV-2 RNA, and if patients were diagnosed in time, the severity of the disease can be effectively predicted and the progression of the disease from mild to severe condition can be reduced, which is of great clinical significance for the pandemic prevention and control of COVID-19. A retrospective cohort study from Wuhan, China, found that the median duration of viral shedding in recovered patients was 20.0 days, and the longest duration of viral shedding was 37.0 days (19). However, some of the infected individuals showed persistent positive nucleic acid test result. A study from Shanghai, China, reported that four COVID-19 cases with persistent positive nucleic acid test had an average conversion time of 61 days from positive to negative nucleic acid test (20). In our study, the median conversion time from the first positive to negative nucleic acid test was 24 days and 25 days in mild and severe patients, respectively, and the conversion time in one of the severe patients was up to 75 days. Zhou et al. found that the duration of viral RNA shedding in the upper respiratory tract specimens from a 75-year-old male patient with COVID-19 reached 111 days (21). Patients infected with SARA-CoV-2 are still shedding virus after six months of treatment, and this is called a chronic carrier state in the human body. The long-term positive viral RNA test result may be due to the damage of the immune system, immune tolerance and escape, or the mutation of the virus $(20,22)$. Further studies are still needed to verify whether COVID-19 will form a chronic carrier state in humans. 
The condition of severe patients with COVID-19 is critical and can change rapidly(23). Our results demonstrated that the white blood cell count, neutrophil count, and lymphocyte count were significantly lower in the severe group than in the mild group, suggesting that early diagnosis and intervention are very important to reduce the risk of death caused by the tendency to severe condition transformation(24). A study comparing the virus shedding time between patients hospitalized in ICU and those not hospitalized in ICU, showed that the duration of virus shedding time of blood, saliva, and nasal samples was longer in ICU patients than in non-ICU patients(25). The reason may be related to the fact that severe patients might be more likely to receive invasive mechanical ventilation $(15,26)$. However, Zheng et al. (27) and Zhou et al. (21) found there was no association between the virus shedding time and the severity of COVID-19. In this study, we divided the virus shedding time into two time periods: from symptom onset to positive nucleic acid test and from positive nucleic acid test to negative nucleic acid test, and the study results indicated that there was no association between the severity of the disease and the time from positive to negative nucleic acid test of the throat swab samples. Further studies revealed that there were no significant differences in the average length of hospital stay between these two groups. The severity of the disease did not affect the conversion time of nucleic acid test and the length of hospital stay, which may be due to the fact that there was no significant positive correlation between the copy number of SARS-CoV-2 viral RNA and the severity of the disease (28). And viral clearance of SARS-CoV-2 may depend mainly on the host's own immune status. Previous studies have shown that advanced age and corticosteroid therapy use are risk factors for prolonged nucleic acid conversion. Elderly COVID-19 patients often show impaired immunity, which reduces the body's ability to clear the virus (29). Glucocorticoid therapy may be effective in suppressing T-lymphocyte-mediated immune responses (4, 30). In clinical practice, some severe COVID-19 patients have a short conversion time from positive to negative nucleic acid test, however, some mild patients and asymptomatic patients with SARS-CoV-2 infection may have a longer conversion time from positive to negative nucleic acid test due to insufficient immune responses(31). Further clinical and scientific researches are still needed to explore the effect of disease severity on the time of nucleic acid conversion of SARS-CoV-2 virus.

\section{Conclusions}

Patients with more severe disease and longer duration of disease before admission to hospitals had a shorter time from symptom onset to positive nucleic acid test. Prolonged time from symptom onset to positive nucleic acid test was an independent risk factor for the prolonged negative conversion time of SARS-CoV-2 virus, and the severity of the disease had no correlation with negative conversion time of SARS-CoV-2 virus.

\section{Abbreviations}

COVID-19: Coronavirus disease 2019; SARS-CoV-2: Severe acute respiratory syndrome coronavirus 2; WHO: World Health Organization; IQR: Interquartile range;

RT-PCR: real-time reverse transcriptase polymerase chain reaction. 


\section{Declarations}

\section{Acknowledgments}

We thank all front-line health-care workers for their joint efforts to fight against SARS-CoV-2. Also, we give especial thanks the staff of the East Campus of the Renmin Hospital of Wuhan University for their kind help with this study.

\section{Authors' contributions}

Yongzhen Zhai and Kaihuan Yu contributed to the conception and design of this research and finally approved the version to be submitted. Xin Zang, Liangkun Xiong, Junyao Zhu and Fangfang Zhao performed data analysis and interpretation and wrote the manuscripts. Liangkun Xiong, Shihong Wang and Wenhui Zeng contributed to data acquisition and inspection.

\section{Funding}

Not applicable.

\section{Availability of data and materials}

The data sets used and analyzed during the current study are available from the corresponding author on reasonable request.

\section{Ethics approval and consent to participate}

This study protocol meets the requirements of the Medical Ethics Committee of Renmin Hospital of Wuhan University. Written informed consent was waived due to the urgency of the COVID-19 pandemic.

\section{Consent for publication}

Not applicable.

\section{Competing interests}

The authors declare that they have no competing interests.

\section{Author details}

${ }^{1}$ Department of Infectious Disease, Shengjing Hospital of China Medical University, Shenyang, 110004, China

${ }^{2}$ Department of Hepatobiliary Surgery, Renmin Hospital of Wuhan University, Wuhan, 430000, China.

${ }^{3}$ Department of Infectious Disease, Fujian Medical University Affiliated First Quanzhou Hospital, Quanzhou, Fujian 362000, China. 
${ }^{4}$ Department of Paediatrics, Renmin Hospital of Wuhan University, Wuhan, 430000, China.

\section{References}

1. Corman VM, Landt O, Kaiser M, et al. Detection of 2019 novel coronavirus (2019-nCoV) by real-time RT-PCR. Euro Surveill. 2020;25(3). doi:10.2807/1560-7917.ES.2020.25.3.2000045.

2. National Health Commission. Diagnosis and Treatment Plan of COVID-19 Trial. 7th ed. China: National Health Commission; 2020. http://www.nhc.gov.cn/xcs/zhengcwj/202003/46c9294a7dfe4cef80dc7f5912eb1989.shtml.

3. Liao T, Yin Z, Xu J, et al. The Correlation Between Clinical Features and Viral RNA Shedding in Outpatients With COVID-19. Open Forum Infect Dis. 2020;7(8):ofaa331. doi:10.1093/ofid/ofaa331.

4. Feng Z, Li J, Yao S, et al. Clinical Factors Associated with Progression and Prolonged Viral Shedding in COVID-19 Patients: A Multicenter Study. Aging Dis. 2020;11(5):1069-81.

doi:10.14336/AD.2020.0630.

5. Yang $Y, H u X$, Xiong L, et al. Clinical characteristics of hospitalized mild/moderate COVID-19 patients with a prolonged negative conversion time of SARS-CoV-2 nucleic acid detection. BMC Infect Dis. 2021;21(1):141. doi:10.1186/s12879-021-05851-z.

6. Campbell $F$, Archer B, Laurenson-Schafer $\mathrm{H}$, et al. Increased transmissibility and global spread of SARS-CoV-2 variants of concern as at June 2021. Euro Surveill. 2021;26(24). doi:10.2807/15607917.ES.2021.26.24.2100509.

7. Choudhary OP, Priyanka, Singh I, Rodriguez-Morales AJ. Second wave of COVID-19 in India: Dissection of the causes and lessons learnt. Travel Med Infect Dis. 2021;43:102126. doi:10.1016/j.tmaid.2021.102126.

8. Li Z, Liu F, Cui J, et al. Comprehensive large-scale nucleic acid-testing strategies support China's sustained containment of COVID-19. Nat Med. 2021;27(5):740-2. doi:10.1038/s41591-021-01308-7.

9. Xing Y, Wong GWK, Ni W, Hu X, Xing Q. Rapid Response to an Outbreak in Qingdao, China. N Engl J Med. 2020;383(23):e129. doi:10.1056/NEJMc2032361.

10. Cao S, Gan Y, Wang C, et al. Post-lockdown SARS-CoV-2 nucleic acid screening in nearly ten million residents of Wuhan, China. Nat Commun. 2020;11(1):5917. doi:10.1038/s41467-020-19802-w.

11. Nolan T, Hands RE, Bustin SA. Quantification of mRNA using real-time RT-PCR. Nat Protoc. 2006;1(3):1559-82. doi:10.1038/nprot.2006.236.

12. Song CY, Yang DG, Lu YQ. A COVID-19 patient with seven consecutive false-negative rRT-PCR results from sputum specimens. Intern Emerg Med. 2020;15(5):871-4. doi:10.1007/s11739-020-02423-y.

13. Li D, Wang D, Dong J, et al. False-Negative Results of Real-Time Reverse-Transcriptase Polymerase Chain Reaction for Severe Acute Respiratory Syndrome Coronavirus 2: Role of Deep-Learning-Based CT Diagnosis and Insights from Two Cases. Korean J Radiol. 2020;21(4):505-8. doi:10.3348/kjr.2020.0146. 
14. West CP, Montori VM, Sampathkumar P. COVID-19 Testing: The Threat of False-Negative Results. Mayo Clin Proc. 2020;95(6):1127-9. doi:10.1016/j.mayocp.2020.04.004.

15. Zheng S, Fan J, Yu F, et al. Viral load dynamics and disease severity in patients infected with SARSCoV-2 in Zhejiang province, China, January-March 2020: retrospective cohort study. BMJ. 2020;369:m1443. doi:10.1136/bmj.m1443.

16. Marks M, Millat-Martinez P, Ouchi D, et al. Transmission of COVID-19 in 282 clusters in Catalonia, Spain: a cohort study. Lancet Infect Dis. 2021;21(5):629-36. doi:10.1016/S1473-3099(20)30985-3.

17. Argyropoulos KV, Serrano A, Hu J, et al. Association of Initial Viral Load in Severe Acute Respiratory Syndrome Coronavirus 2 (SARS-CoV-2) Patients with Outcome and Symptoms. Am J Pathol. 2020;190(9):1881-7. doi:10.1016/j.ajpath.2020.07.001.

18. Li Y, Yang T, Wang S, et al. The value of lymphocyte count in determining the severity of COVID-19 and estimating the time for nucleic acid test results to turn negative. Bosn J Basic Med Sci. 2021;21(2):235-41. doi:10.17305/bjbms.2020.4868.

19. Zhou F, Yu T, Du R, et al. Clinical course and risk factors for mortality of adult inpatients with COVID19 in Wuhan, China: a retrospective cohort study. Lancet. 2020;395(10229):1054-62. doi:10.1016/S0140-6736(20)30566-3.

20. Wei $B$, Hang $X, X i e Y$, et al. Long-term positive severe acute respiratory syndrome coronavirus 2 ribonucleic acid and therapeutic effect of antivirals in patients with coronavirus disease: Case reports. Rev Soc Bras Med Trop. 2020;53:e20200372. doi:10.1590/0037-8682-0372-2020.

21. Zhou C, Zhang T, Ren H, et al. Impact of age on duration of viral RNA shedding in patients with COVID-19. Aging. 2020;12(22):22399-404. doi:10.18632/aging.104114.

22. Ghorbani A, Samarfard S, Ramezani A, et al. Quasi-species nature and differential gene expression of severe acute respiratory syndrome coronavirus 2 and phylogenetic analysis of a novel Iranian strain. Infect Genet Evol. 2020;85:104556. doi:10.1016/j.meegid.2020.104556.

23. Yao T, Gao Y, Cui Q, et al. Clinical characteristics of a group of deaths with COVID-19 pneumonia in Wuhan, China: a retrospective case series. BMC Infect Dis. 2020;20(1):695. doi:10.1186/s12879-02005423-7.

24. Xiong $L$, Zang $X$, Feng G, et al. Clinical characteristics and peripheral immunocyte subsets alteration of 85 COVID-19 deaths. Aging. 2021;13(5):6289-97. doi:10.18632/aging.202819.

25. Fang Z, Zhang Y, Hang C, Ai J, Li S, Zhang W. Comparisons of viral shedding time of SARS-CoV-2 of different samples in ICU and non-ICU patients. J Infect. 2020;81(1):147-78. doi:10.1016/j.jinf.2020.03.013.

26. Lu Y, Li Y, Deng W, et al. Symptomatic Infection is Associated with Prolonged Duration of Viral Shedding in Mild Coronavirus Disease 2019: A Retrospective Study of 110 Children in Wuhan. Pediatr Infect Dis J. 2020;39(7):e95-e9. doi:10.1097/INF.0000000000002729.

27. Zheng X, Chen J, Deng L, et al. Risk factors for the COVID-19 severity and its correlation with viral shedding: A retrospective cohort study. J Med Virol. 2021;93(2):952-61. doi:10.1002/jmv.26367. 
28. To KK, Tsang OT, Leung WS, et al. Temporal profiles of viral load in posterior oropharyngeal saliva samples and serum antibody responses during infection by SARS-CoV-2: an observational cohort study. Lancet Infect Dis. 2020;20(5):565-74. doi:10.1016/S1473-3099(20)30196-1.

29. Yu C, Lei Q, Li W, et al. Clinical Characteristics, Associated Factors, and Predicting COVID-19 Mortality Risk: A Retrospective Study in Wuhan, China. Am J Prev Med. 2020;59(2):168-75. doi:10.1016/j.amepre.2020.05.002.

30. Li T, Cao Z, Chen Y, et al. Duration of SARS-CoV-2 RNA shedding and factors associated with prolonged viral shedding in patients with COVID-19. J Med Virol. 2021;93(1):506-12. doi:10.1002/jmv.26280.

31. Yazdanpanah F, Hamblin MR, Rezaei N. The immune system and COVID-19: Friend or foe? Life Sci. 2020;256:117900. doi:10.1016/j.lfs.2020.117900. 